\title{
A UNIQUE DECOMPOSITION OF INVOLUTIONS OF HANDLEBODIES
}

\author{
ROGER B. NELSON
}

\begin{abstract}
We consider a PL involution of an orientable, 3-dimensional handlebody for which each component of the fixed point set is 2-dimensional. The handlebody is uniquely equivariantly decomposed as a disk sum of handlebodies $M_{i}$ such that if $M_{i} \approx A_{i} \times I$, then $h \mid M_{i}$ is equivalent to (i) $\alpha \times \mathrm{id}_{I}$, where $\alpha$ is an involution of $A$, or to (ii) id ${ }_{a} \times r$, where $r(t)=-t$ for all $t \in I=[-1,1]$.
\end{abstract}

1. Introduction. In this paper all spaces and all mappings are objects in the piecewise linear (PL) category. Let $M_{i}$ be a bordered 3-manifold and let $h_{i}$ be an involution of $M_{i}, i=1,2$. We define $h_{1}$ as equivalent to $h_{2}$, denoted $h_{1} \sim h_{2}$, if there exists a homeomorphism $f: M_{1} \rightarrow M_{2}$ such that $f \circ h_{1}=h_{2} \circ f$, i.e. $h_{1}$ and $h_{2}$ are conjugate in the group of homeomorphisms on $M$. We call $h$ a type (i) involution of $M$ if $M \approx A \times I$, where $A$ is a 2-manifold and $I=[-1,1]$, and if $h \sim \alpha \times \mathrm{id}_{I}$, where $\alpha$ is an involution of $A . h$ is said to be a type (ii) involution if $h \sim \mathrm{id}_{A} \times r$, where $r(t)=-t$ for all $t \in I$. In this paper we show that, under suitable conditions, a handlebody $M$ is uniquely equivariantly decomposed as $M=M^{\prime} \cup\left(\cup_{i=1}^{n} T_{i}\right)$, where $h \mid M^{\prime}$ is a type (i) involution and each $h \mid T_{i}$ is a type (ii) involution.

This paper is concerned only with involutions $h: M \rightarrow M$, where each component of the fixed point set of $h, F(h)$, is 2-dimensional and orientable and $M$ is an orientable, 3-dimensional handlebody. Recall that a handlebody of genus $n$ is a space obtained by choosing $2 n$ disjoint disks in the boundary of a 3-ball and identifying them in pairs by $n$ PL homeomorphisms. Two handlebodies are homeomorphic if and only if they have the same genus and are both orientable or both nonorientable.

In [2] it was proved that such an involution $h$ is type (i) if and only if $F(h)$ is a collection of disks and annuli. It was also shown that $h$ is type (ii) if and only if $F(h)$ is orientable, connected and $\chi(F(h))=\chi(M)$. It is the purpose of this paper to show that these type (i) and type (ii) involutions are the building blocks for all involutions with orientable, 2-dimensional fixed point sets on orientable handlebodies. In particular, we will prove

THEOREM A. Let $M$ be an orientable handlebody with an involution $h$ having orientable 2-dimensional fixed point set. Then $M=M^{\prime} \cup\left(\cup_{i=1}^{n} T_{i}\right)$, where $h\left(M^{\prime}\right)=$ $M^{\prime}, h\left(T_{i}\right)=T_{i}, M^{\prime} \cap T_{i}$ is a disk for each $i$, and $T_{i} \cap T_{j}=\varnothing$ if $i \neq j$. Furthermore, $h \mid M^{\prime}$ is type (i) and $h \mid T$ is type (ii) for each $i$.

Received by the editors September 14, 1982 and, in revised form, September 7, 1983 and April 4, 1984. 1980 Mathematics Subject Classification. Primary 57Q99, 57S17, 57S25.

Key words and phrases. Involution, equivalent involutions, equivariant decompositions of handlebodies, 2-dimensional fixed point set, invariant properly embedded disks. 
THEOREM B. If $M=M^{\prime} \cup\left(\bigcup_{i=1}^{n} T_{i}\right)$ and $M=M^{\prime \prime} \cup\left(\bigcup_{i=1}^{n} T_{i}^{\prime}\right)$ are two decompositions of the involution $h$ on $M$ of the type obtained in Theorem A, then $h\left|M^{\prime} \sim h\right| M^{\prime \prime}$ and $h\left|T_{i} \sim h\right| T_{i}^{\prime}$ for all $i$ after a suitable reordering.

Technical lemmas are provided in $\$ 2$, while the decomposition and uniqueness theorems are proved in $\S 3$. In $\$ 4$ we show that inequivalent involutions may not have equivalent decompositions in the sense of Theorem $B$.

\section{Technical lemmas.}

LEMMA 2.1. Let $M$ be an orientable handlebody with involution $h$ having 2-dimensional fixed point set $F(h)$ containing a nondisk component. Then there exists a properly embedded, invariant disk $D$ such that $D$ is nonseparating and cutting along $D$ lowers the genus of $M$ and also lowers the genus of some component of $F(h)$.

Proof. [2, Lemma 2.3] provides an invariant disk $D^{\prime}$ which either satisfies our criteria or else separates $M$ into two nontrivial components. A simple induction on $g(M)$, the genus of $M$, will show that $D^{\prime}$ can always be chosen to meet our criteria. When $g(M)=1, M$ is a solid torus which by [1] cannot be decomposed into two nontrivial components.

Suppose $g(M)>1$. If the disk $D^{\prime}$ provided by [2, Lemma 2.3] does not satisfy the criteria, then we may write $M$ as a disk sum $M=M_{1} \Delta M_{2}$, where $h\left(M_{i}\right)=M_{i}$, $i=1,2$. One of these components, say $M_{1}$, has a nondisk component of $F\left(h \mid M_{1}\right)$ and $g\left(M_{1}\right)<g(M)$. Hence, by induction hypothesis, $M_{1}$ contains an invariant, properly embedded disk $D$ meeting the criteria. If $D \cap D^{\prime}=\varnothing$, then $D$ is the desired disk in $M$. It remains only to show that $D$ may be chosen so that $D \cap D^{\prime}=\varnothing$.

Suppose $D \cap D^{\prime} \neq \varnothing$. Let the simple closed curve $D \cap \partial M_{1}$ be called $c$. Then $c \cap D^{\prime}$ could conceivably be either a simple closed curve or a union of arcs. If $c \cap D^{\prime}$ is a simple closed curve, then $D$ is boundary parallel in $M_{1}$, which violates the choice of $D$. Hence $c \cap D^{\prime}$ is a union of arcs. Let $\alpha$ be any $\operatorname{arc}$ in $c \cap D^{\prime}$. If $p$ is the projection map of $M_{1}$ onto its orbit space $M_{1}^{*}$, then $p(D) \cap p\left(D^{\prime}\right)$ contains $p(\alpha)$, an arc on $\partial\left(M_{1}^{*}\right)$. Let $H$ be an isotopy of $\partial\left(M_{1}^{*}\right)$ which moves $p(\alpha)$ off $p\left(D^{\prime}\right)$, is constant in a small regular neighborhood of $p\left(D^{\prime}\right)$ in $\partial\left(M_{1}^{*}\right)$, and such that $H_{t}\left(p\left(F(h) \cap M_{1}\right)\right)=p\left(F(h) \cap M_{1}\right) \subset \partial\left(M_{1}^{*}\right)$. Since $\partial\left(M_{1}^{*}\right)$ is compact, it is collared [3, Theorem 2.26], so $H$ lifts to an isotopy $G$ of $M$ which commutes with $h \mid M_{1}$. Then $G_{1}(D)$ is a properly embedded, invariant disk meeting the desired criteria and with $G_{1}(D) \cap D^{\prime} \subset D \cap D^{*}-\{\alpha\}$. Repeating this procedure for each arc in $D \cap D^{\prime}$ yields the desired disk having null intersection with $D^{\prime}$.

REMARK 2.1.1. The argument of the last paragraph applied to $M$ rather than $M^{\prime}$ allows us to assume that $D$ can be chosen to have null intersection with any finite collection of invariant disks on $\partial M$.

LEMMA 2.2. Suppose $M$ is an orientable, 3-dimensional handlebody with involution $h$ having orientable, 2-dimensional fixed point set $F(h)$, and $M$ contains a properly embedded, h-invariant disk $D$ which intersects two distinct boundary components of $F(h)$. Then $M=M^{\prime} \Delta T$, where $h\left(M^{\prime}\right) \Delta T$, where $h\left(M^{\prime}\right)=M^{\prime}, h(T)=T, T$ is a solid torus and $M^{\prime} \approx M-N(D)$ is a handlebody of genus one less than $M$. 


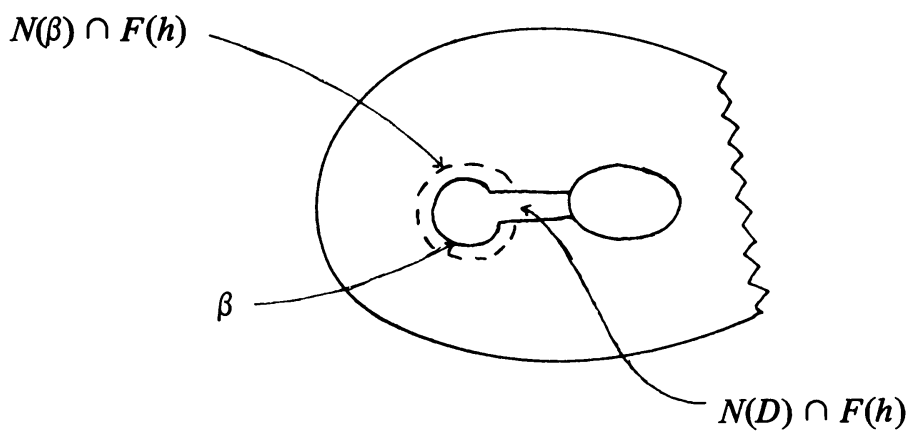

FIGURE 1

Proof. Let $M^{\prime \prime}=\overline{M-N(D)}$, where $N(D)$ is a small, invariant, regular neighborhood of $D$ in $M . N(D) \simeq D \times I$ and we let $D_{0}=D \times\{0\}$ and $D_{1}=D \times\{1\}$. Then $D_{0} \cap F\left(h \mid M^{\prime \prime}\right)=\alpha_{0}$ and $D_{1} \cap F\left(h \mid M^{\prime \prime}\right)=\alpha_{1}$ are $\operatorname{arcs}$ on $\partial F\left(h \mid M^{\prime \prime}\right)$. If $\alpha_{0}$ and $\alpha_{1}$ are viewed as arcs in $F(h)$, they each connect the same two components of $\partial F(h)$. Let $C$ be one of the boundary curves of $F(h)$ met by $\alpha_{0}$ and $\alpha_{1}$. Let the $\operatorname{arc} C-N(D)$ be called $\beta$ (see Figure 1).

We view $\beta$ as an arc in $\partial\left(M^{\prime \prime}\right)$. If $N(\beta)$ is a small, invariant, regular neighborhood in $M^{\prime \prime}$, then $N(\beta) \cap \partial\left(M^{\prime \prime}\right)=D_{2}$ is a disk and $N(\beta) \cup N(D)=T$ is a solid torus. Now $M=M^{\prime \prime} \cup N(D)=M^{\prime} \cup T$, where $M^{\prime}=\overline{M^{\prime \prime}-T}$ and $M^{\prime} \cap T=D_{0} \cup D_{1}$ $\cup D_{2}$, a disk. Hence $M=M^{\prime} \Delta T$ with $h\left(M^{\prime}\right)=M^{\prime}$ and $h(T)=T$. It follows from the unique decomposition of 3-manifolds with connected boundary into $\Delta$-prime manifolds [1] that $M^{\prime}$ is an orientable 3-dimensional handlebody of genus one less than $M$.

REMARK 2.2.1. If disk $D^{\prime} \subset M^{\prime \prime}$ is the scar of some previous cut and $\partial T \cap D^{\prime} \neq 0$, then we may assume that either $\partial T \cap D^{\prime}=\varnothing$ or $D^{\prime} \subset \partial T$. We need only make three quick observations. Since $T$ is a small regular neighborhood of $\beta$, we can presume that $D^{\prime} \cap \beta \neq \varnothing$. Since any "scar" meets $F(h)$ transversely in a single arc and $\beta \subset F(h)$, then $D^{\prime}$ meets $\beta$ in a single arc. Hence, we may replace $N(\beta)$ by $N\left(\beta \cap D^{\prime}\right)$.

\section{The decomposition.}

Proof of Theorem A. An invariant handle can be detached from the handlebody $M$ in either of two ways. By Lemma 2.1, $M$ may be regarded as $M=M^{\prime \prime} \cup N(D)$, where the ball $N(D)$ is a small, invariant, regular neighborhood of the properly embedded disk $D$, and $M^{\prime \prime} \cap N(D)=D_{0} \cup D_{1}$, two disjoint disks. If $D$ intersects distinct components of $\partial F(h)$, then Lemma 2.2 yields $M$ as $M=M^{\prime} \Delta T$, where $T$ is a solid torus and $M^{\prime} \approx M^{\prime \prime}$ is a handlebody with genus one less than that of $M$.

We now successively cut $M$ along disks obtained via Lemma 2.1 or 2.2 which meet nondisk components of $F(h)$. This cutting process is carried out until all nondisk components of $F(h)$ have been reduced to disks. Label the nondisk components of $F(h)$ by $C_{i}, i=1, \ldots, n$. Each time an invariant handle is cut from 
$M$, the remaining portion of exactly one $C_{i}$ is intersected. Let $T_{i, j}$ be the $j$ th ball or torus split from $M$ intersecting the residue of $C_{i}$. Denote the residue of $C_{i}$ after $j$ cuts intersecting $C_{i}$ by $C_{i, j}$.

We now claim that each $T_{i, j}$ can be chosen that the "scar" of the cut creating $T_{i, j}$ is on some $T_{i, k}$ for $k>j$. If $T_{i, k}$ intersects the scar of $T_{i, j}$ for some $k>j$ it may be assumed to contain it by Remark 2.2.1. To establish the claim, therefore, it is only necessary to show that the last cut from $C_{i}$, the torus $T_{i, n_{i}}$, can be accomplished so that $\partial T_{i, n_{i}}$ contains the scar of each $T_{i, j}$ whose scar is not contained on some $T_{i, k}$ for some $k<n_{i}$. This is done by augmenting the solid torus $T_{i, n_{i}}$ provided by Lemma 2.2 and Remark 2.2 .1 by an appropriately chosen ball $B$. $B$ is an invariant, regular neighborhood in the remainder of $M$ and the set $k$ consisting of a carefully chosen arc on $\partial\left(C_{i, n_{i-1}}\right)$ and all the scars it intersects. $\alpha$ is chosen to intersect all scars not on $T_{i, k}$ for some $k<n_{i}$ or on $B$ (see Figure 2).

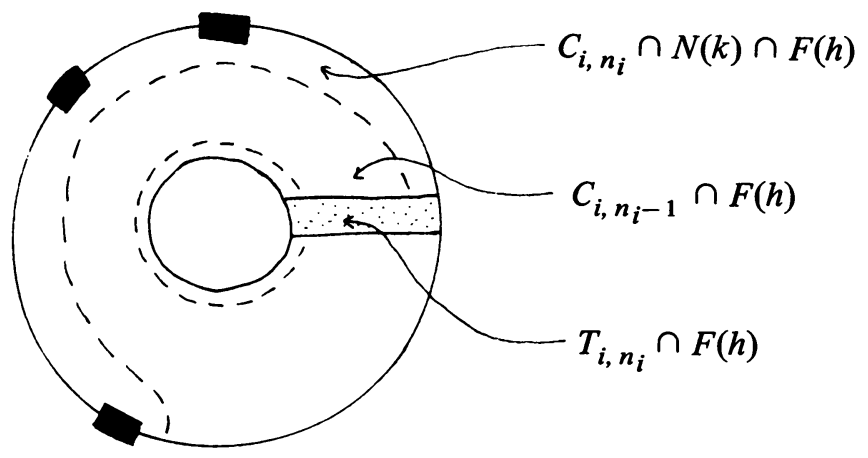

FIGURE 2

Now define $T_{i}=\bigcup_{i=1}^{n} T_{i, j}$. Then $M=M^{\prime} \bigcup_{i=1}^{n} T_{i}$, where $T_{i} \cap T_{k}=\varnothing$ if $i \neq k$ and $M^{\prime} \cap T_{i}=M^{\prime} \cap T_{i, n_{i}}$ is a disk for each $i$. Clearly $F\left(h \mid M^{\prime}\right)$ is a collection of disks, so $h \mid M^{\prime}$ is type (i). It remains to show that $h \mid T_{i}$ is type (ii) for each $i$.

The argument that $h \mid T_{i}$ is type (ii) has three parts. First we note that

$$
\chi\left(F(h) \cap T_{i, j}\right)=\chi\left(T_{i, j}\right) .
$$

This follows immediately from the facts that either $T_{i, j}=N(\beta) \cup N(D)$, where $N(\beta)$ and $N(D)$ are balls whose intersection consists of two disks, and that $T_{i, j} \cap F(h)=(N(\beta) \cap F(h)) \cup(N(D) \cap F(h))$, where $N(\beta) \cap F(h)$ and $N(D) \cap$ $F(h)$ are disks intersecting in two arcs, or that $T_{i, j}$ is a ball and $T_{i, j} \cap F(h)$ is a disk.

Second, we claim that $\chi\left(T_{i}\right)=\chi\left(T_{i} \cap F(h)\right)$. Let $T_{i}^{k}$ be a partial reconstruction of $T_{i}$ from $\left\{T_{i, j} \mid j=1, n_{i}\right\}$ given by $T_{i}^{k}=\bigcup_{j=i}^{k} T_{i, n_{i}-j+1}$. We just reconstruct $T_{i}$ in the reverse order from which we picked it apart. Then $T^{1}=T_{i, n_{i}}, T_{i}^{n_{i}}=T_{i}$ and $T_{1}^{k}=$ $T_{i}^{k-1} \cup T_{i, n_{i}-k+1}$, where the scar of $T_{i, n_{i}-k+1}$ is on some $T_{i, n_{i}-l+i} \subset T_{i}^{k+1}, l<k$ (see the third paragraph of this section). The claim then follows immediately by finite induction on $k$.

Finally, we appeal to [2, Theorem 3.2] to show that $h \mid T_{i}$ is type (ii), having shown that $T_{i} \cap F(h)=F\left(h \mid T_{i}\right)$ is a connected, orientable, bordered surface with

$$
\chi\left(T_{i} \cap F(h)\right)=\chi\left(T_{i}\right) .
$$


Proof of Theorem B. A decomposition is of little value, of course, unless it is in some sense unique. The uniqueness of this decomposition follows with unusual ease. Observe that $C_{i} \simeq T_{i} \cap F(h)=F\left(h \mid T_{i}\right)$ and that $T_{i} \simeq C_{i} \times I$ since $h \mid T_{i}$ is type (ii). Hence the collections $\left\{T_{i}\right\}$ and $\left\{h \mid T_{i}\right\}$ are uniquely determined up to equivalence and order by the nondisk components of $F(h)$.

Note, also, that $M^{\prime} \cap C_{i}=\overline{C_{i}-\left(C_{i} \cap T_{i}\right)}$ is a disk for each $i$. Therefore, if $F(h)$ consists of $n$ nondisk components and $m$ disks, then $F\left(h \mid M^{\prime}\right)$ consists of $m+n$ disks. Furthermore, $F\left(h \mid M^{\prime}\right)$ separates $M^{\prime}$ if and only if $F(h)$ separates $M$, and $g\left(M^{\prime}\right)=g(M)-\sum_{i=1}^{n} g\left(T_{i}\right)$. Hence, $M^{\prime}$ and $h \mid M^{\prime}$ are uniquely determined up to equivalence [2, Theorem 4.1]. Therefore, this decomposition is unique in the sense of Theorem B.

4. We must finally address the question of whether inequivalent involutions can possess equivalent (in the sense of Theorem B) unique decompositions. We will find a negative answer. If $h$ and $g$ are involutions on $M$ and $N$, respectively, they have equivalent decompositions in the sense of Theorem B if and only if $g(M)=g(N)$, $F(h) \simeq F(g)$ and both $F(h)$ and $F(g)$ separate or both do not separate. If $h$ and $g$ are type (i) or type (ii) involutions, then [2, Theorem 4.1] indicates that $h \sim g$ if these three conditions hold. This result is easily generalized to the broader class of involutions considered here.

THEOREM C. Let $h$ and $g$ be involutions on orientable, 3-dimensional handlebodies $M$ and $N$ such that the fixed point sets $F(h)$ and $F(g)$ are 2-dimensional. Then $h \sim g$ if and only if $g(M)=g(N), F(h) \simeq F(g)$ and either $F(h)$ and $F(g)$ both separate or both do not separate.

Proof. The proof is basically an induction on $g(M)=g(N)$. If $g(M)=1$, then $M$ and $N$ are solid tori and $h$ and $g$ are both type (i) or type (ii) so that the result holds. Suppose $g(M)>1$ and $f$ and $g$ are not type (i) or type (ii). Let $M$ and $N$ be decomposed as $M=M^{\prime} \cup\left(\cup_{i=1}^{n} T_{i}\right)$ and $N=N^{\prime} \cup\left(\cup_{i=1}^{n} T_{i}^{\prime}\right)$. Pick $T_{1}$ and $T_{1}^{\prime}$ so that $T_{1} \simeq T_{1}^{\prime}$. Since $H\left|T_{1} \simeq g\right| T_{1}^{\prime}$ are type (ii) we can easily obtain invariant, properly embedded disks $D \subset T_{1}$ and $D^{\prime} \subset T_{1}^{\prime}$. Let $M_{1}=M-N(D)$ and $N_{1}=N$ $-N\left(D^{\prime}\right)$. Then $h\left|M_{1} \sim g\right| N_{1}$ by the induction hypothesis. The remainder of the induction follows exactly as in [2, Theorem 4.1].

\section{BIBLIOGRAPHY}

1. J. L. Gross, $A$ unique decomposition theorem for 3-manifolds with connected boundary, Trans. Amer. Math. Soc. 142 (1969), 191-199.

2. R. B. Nelson, Some fiber preserving involutions of orientable 3-dimensional handlebodies, Houston J. Math. 9 (1983), 255-269.

3. C. P. Rourke and B. J. Sanderson, Introduction to piecewise-linear topology, Springer-Verlag, Berlin, 1972.

Department of Mathematical Sciences, Ball State University, Muncie, Indiana 47306 